\title{
Heat transfer study on a hybrid smooth and spirally corrugated tube
}

\author{
Chen Yang ${ }^{1}$, Min-rui Chen ${ }^{1}$, Jin-yuan Qian ${ }^{1,2, *}$, Zan $\mathrm{Wu}^{2}$, Zhi-jiang Jin ${ }^{1}$, and Bengt Sunden ${ }^{2}$ \\ ${ }^{1}$ Institute of Process Equipment, College of Energy Engineering, Zhejiang University, Hangzhou, 310027, PR China \\ ${ }^{2}$ Department of Energy Sciences, Lund University, P.O. Box 118, SE-22100 Lund, Sweden
}

\begin{abstract}
Corrugated tubes are widely used in a range of applications for heat transfer enhancement. The spirally corrugated tube has a better heat transfer performance than the smooth tube. In this paper, the heat transfer performance of a hybrid smooth and six-start spirally corrugated tube is studied. With a validated numerical model, the effects of the corrugation part length on the vortex in the downstream smooth tube are studied for a range of high Reynolds numbers, where the existence of the corrugation part can turn out the secondary flow and enhance heat transfer. Meanwhile, it is found that in the smooth part, the fluid flow part with whirling can reach a maximum length, even if the length of the corrugation part continuously increases. Thus a series of critical corrugation lengths can be obtained. This work can reveal the enhanced heat transfer mechanism of the hybrid smooth and spirally corrugated tube and be of interest to researchers in heat transfer issues of corrugated tubes.
\end{abstract}

\section{Introduction}

Spirally corrugated tubes, owning the merits of better thermal performance, have been widely used in coaxial heat exchangers and have attracted the attention of researchers on heat transfer characteristics.

Mimura and Iaozaki [1] compared the corrugated tubes with smooth tubes on heat transfer and pressure drop performance under the effects of spiral structures. Others confirmed the influence spiral corrugation exerts on enhancing heat transfer performance through experimental works and numerical simulation [2-7]. Taking account of corrugation depth (DR), pitch ratio (PR) and Reynolds number, Promthaisong et al. [8] discovered that heat transfer enhancement was induced by vortex flows generated only around the wall corrugation.

Nowadays, a wide range of researchers have turned their research priority to multi-start spirally corrugated tubes given their exclusive structure and good heat transfer performance. Lazim et al. [9] presented a numerical analysis on thermal characteristics and pressure drop performance. Taking four-start spirally corrugated tubes as an example, they concluded that more corrugations can enhance flow mixing. Kareem et al. [10-11] performed numerical simulation on two-start and three-start spirally corrugated tubes and indicated that corrugation profiles determined thermal performance, which had a better effect at high Re. Balla
[12] conducted a numerical study and studied the effects of corrugation characteristics on heat transfer performance. Jin et al. [13-14] also investigated six-start spirally corrugated tubes and obtained a criterion correlation for flow resistance as well as heat transfer characteristics. Sun et al. [15] and Vocale et al. [16] applied the field synergy theory to analyse the augmentation effect. Promthaisong et al. [17] made a conclusion that main swirl flow and five-secondary swirl flow enhanced heat transfer process of five-start spiral corrugated tube.

\section{Analysis and modelling}

\subsection{Geometric model}

The copper smooth tube experiences a rolling operation in a rotational stretching way, passing through six processing mechanisms successively. As a result, the structure of six-start spirally corrugated tube is shown as Fig. 1, including geometric parameters.

\footnotetext{
Corresponding author: qianjy@zju.edu.cn
} 


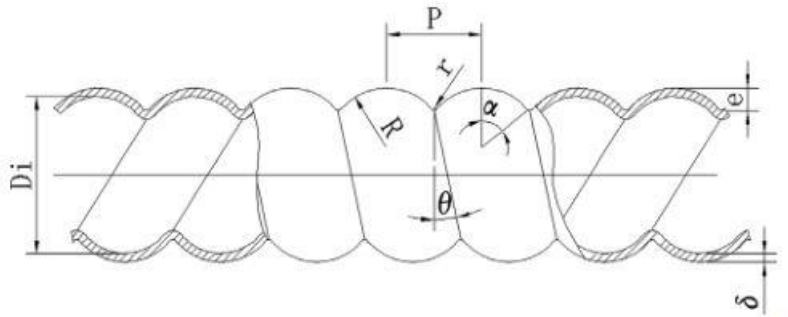

Fig. 1. Structural parameters of six-start spirally corrugated tube.

To exclude the influence of spiral corrugation parameters, including inter diameter $D_{i}$, pitch $p$ and corrugation depth $e$, we set these variables as constant value, which is elaborated in Table 1.

Table 1. Constant values of variables.

\begin{tabular}{|c|c|c|c|}
\hline $\begin{array}{c}\text { Inter } \\
\text { diameter } \\
\boldsymbol{D}_{\boldsymbol{i}}(\mathbf{m m})\end{array}$ & Pitch $\boldsymbol{p}(\mathbf{m m})$ & $\begin{array}{c}\text { Corrugation } \\
\text { depth } \boldsymbol{e}(\mathbf{m m})\end{array}$ & $\begin{array}{c}\text { Reynolds } \\
\text { number } \boldsymbol{R} \boldsymbol{e}\end{array}$ \\
\hline 27 & 60 & 4.5 & 10000 \\
\hline
\end{tabular}

The hybrid tubes we studied are consisted of six-head spirally corrugated tube and smooth tube. They start from corrugation part, the length of which differs from each other, while the whole length is $1 \mathrm{~m}$. The flow path model can be seen from Fig. 2.

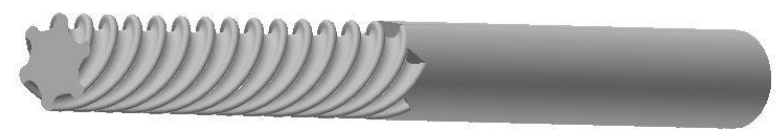

Fig. 2. Flow path model of the hybrid tube.

\subsection{Mesh and boundary condition}

To ensure the accuracy and reliability of solving results, it is of utter necessity to verify the grid independence. We adopt tetrahedral mesh in corrugation part while multi-zone method in smooth part. Furthermore, boundary layer mesh is applied. Eventually, the grid size is controlled in $1 \mathrm{~mm}^{3}$ when the Nusselt number tends to reach its asymptotic value. When grid numbers increases from 3246214 to 3460198 , difference of $N u$ is between the acceptable range of $5 \%$. Simultaneously, the results of assessed $\mathrm{y}+$ coincide with the recommended values ranging from 30 to 100 .

The heat transfer medium in the hybrid smooth and six-start spirally corrugated tube is water. We set inlet boundary as velocity inlet, the temperature of which is $363 \mathrm{~K}$ while outlet boundary as pressure outlet, the value is $0 \mathrm{~Pa}$. The Realizable $k$ - $\varepsilon$ turbulence model, combined with standard wall function method is adopted in the simulation, applying pressure implicit solver, SIMPLE algorithm and the second order upwind scheme. The values of residuals in the convergence conditions are less than $1 \times 10^{-6}$.
Compare the calculated Nusselt number with the value of the empirical formula at $R e$ ranging from $1 \times 10^{4}$ to $2.5 \times 10^{4}$ in smooth tube, the errors are in the allowable range and the largest one is $+4.63 \%$.

\section{Results and discussion}

\subsection{Heat transfer performance}

In this paper, we research the velocity field distribution of a hybrid smooth and six-start spirally corrugated tube.

On the basis of the previous studies, the successive corrugation structure can induce a significant degree of swirl perpendicular to the mainstream direction. As a result, the secondary flow velocity $V_{x y}$ is boosted and heat transfer is enhanced. To figure out the influence, the lengths of the corrugation part are $100 \mathrm{~mm}, 200 \mathrm{~mm}, 300$ $\mathrm{mm}$, corresponding to Tube 1 , Tube 2 and Tube 3 , respectively. The temperatures at various positions of these three tubes, together with the smooth tube are calculated to analyse the heat transfer performance, which is depicted in Fig. 3.

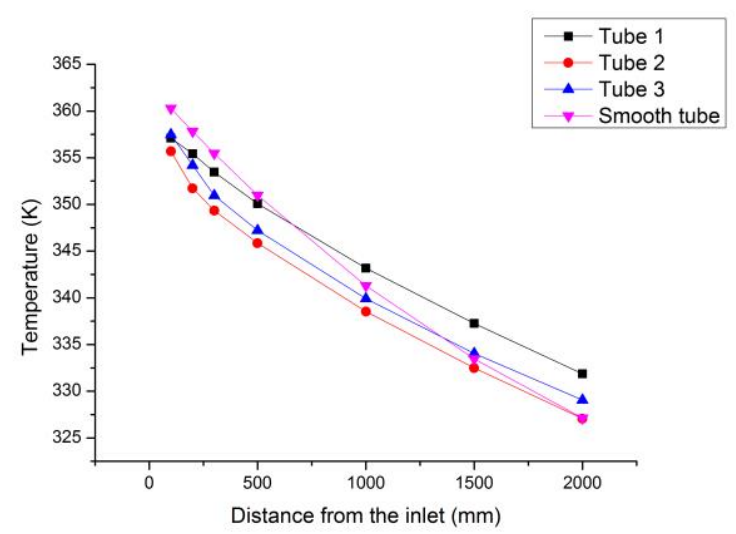

Fig. 3. Temperatures at various positions of four tubes.

\subsection{Velocity analysis}

For further investigation of the flow situation, cross sections of tube 1 are created in $\mathrm{z}=0.01,0.05,0.1 \mathrm{~m}$ (the end of the corrugation part). As is shown in Fig. 4, the velocity contour reflects the secondary flow velocity distribution that fluid of low velocity is around the center position while high at the corrugation. The secondary flow derives from the flowing fluid under the influence of the centrifugal force. The different centrifugal force values result from the variance in axial velocity. Fluid particles flowing in the tube core and close to the tube wall generate the velocity disparity. The former have a higher axial velocity and thus experience a larger centrifugal force. As a result, fluid in the tube core region is pushed towards the outside wall of the tube, where it drives the fluid towards the inside wall of the tube.

\subsection{Reliable verification}




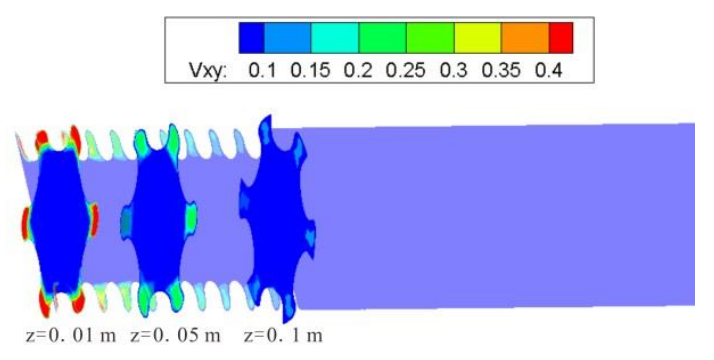

Fig. 4. Secondary flow velocity distribution in $100 \mathrm{~mm}$ corrugation part.

The scope of the centered fluid shrinks as the fluid flows away from the inlet, and the secondary flow at the corrugation is becoming less intensive at the same time. In other words, the secondary flow at the cross section tends to reduce velocity variance, which can be seen in Fig. 5.

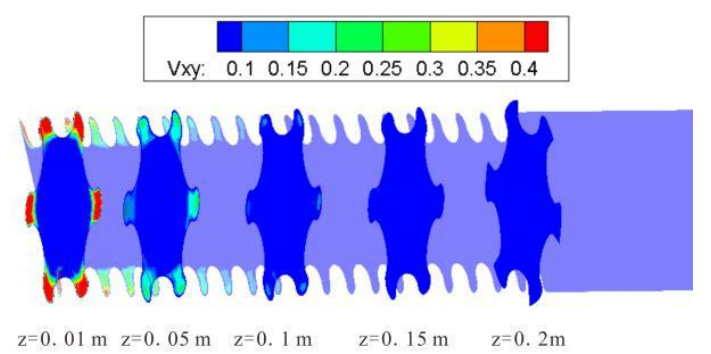

Fig. 5. Secondary flow velocity distribution in $200 \mathrm{~mm}$ corrugation part.

In tube 2 , cross sections are generated in $\mathrm{z}=0.15$ and $0.2 \mathrm{~m}$. Compared with Fig. 6, it can be concluded that the secondary flow keeps developing at the corrugation part. The cross section of tube 3 in $\mathrm{z}=0.25 \mathrm{~m}$ is illustrated in Fig. 6, and the velocity contours almost remain constant, which means the secondary flow gets fully developed $0.2 \mathrm{~m}$ away from the inlet.

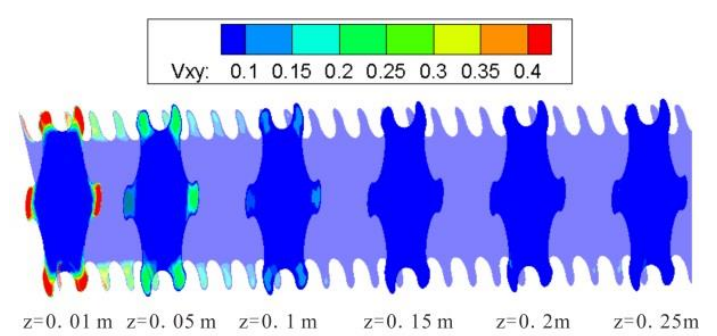

Fig. 6. Secondary flow velocity distribution in $300 \mathrm{~mm}$ corrugation part.

The spirally corrugated tube plays a significant role in generating secondary flow, which gets diminished in smooth tube. The distance from the interface between the corrugation part and smooth part to where secondary flow gets fully vanished is recorded. When the cross section is $0.01 \mathrm{~m}$ away from the interface, the secondary flow velocity vectors are intensive and the fluid particles around the wall are faster than the other part. As the fluid flows through the smooth tube, the secondary velocity vectors tends to distribute evenly and the velocity values of the whole cross section gets slower. As is shown in
Fig. 7, the vector distribution characteristics of three tubes are comparable under the same working condition, in other words, secondary flow dies away following a specific regular pattern even if the length of the corrugation part continuously increases.

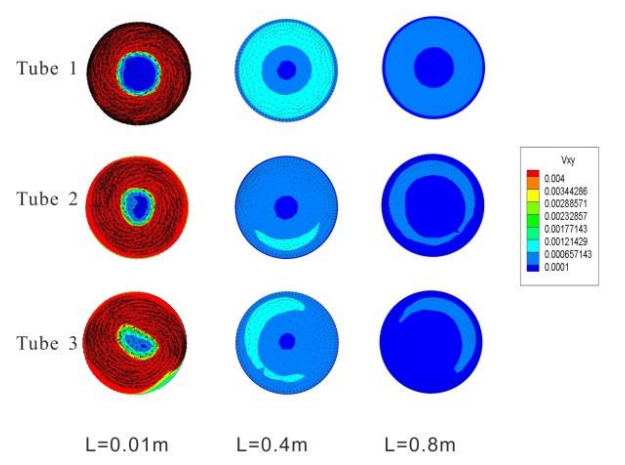

Fig. 7. Comparison of three tubes at same distance from the interface.

No matter how long the corrugation part is, the secondary flow vanishes at the position of $0.8 \mathrm{~m}$ away from the interface.

\section{Conclusion}

In this study, a novel structure hybrid smooth and sixstart spirally corrugated tube is proposed, in order to discover the appropriate length of the corrugation part. Simulation is conducted with fixed geometric parameters under $R e=10,000$. The main conclusions are drawn as follows:

Firstly, the corrugation structure gives rise to the generation of secondary flow and enhances heat transfer as a result. Besides, once the corrugation part is substituted by smooth tube, the secondary flow will experience attenuation and finally vanishes no matter how long the corrugation part is or whether the secondary flow has been fully developed. The key to maintaining secondary flow is the corrugation structure.

This work is supported by the Key project of Natural Science Foundation of Zhejiang Province, China through Grant No. LZ17E050002, and the Fundamental Research Funds for the Central Universities through Grant No. 2018QNA4013.

\section{References}

1. K. Mimura, A. Isozaki, Desalination, 22, 1, pp. 131139, (1977)

2. S. Garimella, R. N. Christensen, SHAAE Trans, 99, 1, pp. 1205-1216, (1993)

3. J.E. Walker, G.A. Whan, R.R. Rothfus, AIChE J., 3, 4, pp. 484-489, (1957)

4. H.M. Li, K.S. Ye, Y.K. Tan, S.J. Deng, Heat Transf, 25, 63, pp. 75-80, (1982)

5. S. Garimella, V. Chandrachood, R.N. Christensen, D. E. Richards, ASHRAE T., 94, pp. 1119-1131, (1988) 
6. T.S.Ravigururajan, A.E. Bergles, J. Heat Transf., 116, 1, pp. 54-57, (1994)

7. Y.X. Li, J.H. Wu, H. Wang, L.P. Kou, X.H. Tian, Energy Proc., 17(Part A), pp. 791-800, (2012)

8. P. Promthaisong, W. Jedsadaratanachai, S. EiamsaArd, Numer. Heat Tranf. A-Appl., 69, 6, pp. 607629, (2016)

9. T.M. Lazim, Z.S. Kareem, M.N.M. Jaafar, S. Abdullah, A.F. Abdulwahid, Int. Rev. Modell. Simul., 7, 6, pp. 970, (2014)

10. Z.S. Kareem, M.N.M. Jaafar., T.M. Lazim, S. Abdullah, A.F. Abdulwahid, Alexandria Eng. J., 54, 3, pp. 415-422, (2015)

11. Z.S. Kareem, S. Abdullah, T.M. Lazim, M.N.M. Jaafar, A.F. Abdulwahid, Chem. Eng. Sci., 134, pp. 746-757, (2015)

12. H.H. Balla, Case Stud. Therm. Eng., 9(C), pp. 79-89, (2017)

13. Z.J. Jin, B.Z. Liu, F.Q. Chen, Z.X. Gao, X.F. Gao, J.Y. Qian, Int. J. Heat Mass Transf., 103, pp. 11981207, (2016)

14. Z.J. Jin, F.Q. Chen , Z.X. Gao , X.F. Gao , J.Y. Qian, Int. J. Heat Mass Transf., 108, pp. 1011-1025, (2017)

15. M. Sun, M. Zeng, Appl. Therm. Eng., 129, pp. 1-11, (2018)

16. P. Vocale, F. Bozzoli, A. Mocerio, S. Rainieri, ICHMT 9th, Napoli, Italy. May 28-June 1, (2017)

17. P. Promthaisong, W. Jedsadaratanachai, V. Chuwattanakul, S. Eiamsa-Ard, Int. Conf. Chem. Mater. Process, pp.020005, (2017) 